\title{
Isotopic Dependence of the Caloric Curve
}

W. Trautmann, ${ }^{1}$ P. Adrich, ${ }^{1}$ T. Aumann, ${ }^{1}$ C.O. Bacri,${ }^{2}$ T. Barczyk, ${ }^{3}$ R. Bassini, ${ }^{4}$ S. Bianchin, ${ }^{1}$ C. Boiano, ${ }^{4}$ A.S. Botvina, ${ }^{1,5}$ A. Boudard, ${ }^{6}$ J. Brzychczyk, ${ }^{3}$ A. Chbihi, ${ }^{7}$ J. Cibor, ${ }^{8}$ B. Czech, ${ }^{8}$ M. De Napoli, ${ }^{9}$ J.-É. Ducret, ${ }^{6}$ H. Emling, ${ }^{1}$ J.D. Frankland, ${ }^{7}$ M. Hellström, ${ }^{1}$ D. Henzlova, ${ }^{1}$ G. Immè ${ }^{9}{ }^{\text {I. Iori }},{ }^{4}$ H. Johansson,${ }^{1}$ K. Kezzar, ${ }^{1}$ A. Lafriakh, ${ }^{6}$ A. Le Fèvre, ${ }^{1}$ E. Le Gentil, ${ }^{6}$ Y. Leifels, ${ }^{1}$ J. Lühning, ${ }^{1}$ J. Eukasik, ${ }^{1,8}$ W.G. Lynch, ${ }^{10}$ U. Lynen, ${ }^{1}$ Z. Majka, ${ }^{3}$ M. Mocko, ${ }^{10}$ W.F.J. Müller, ${ }^{1}$ A. Mykulyak, ${ }^{1}$ H. Orth,${ }^{1}$ A.N. Otte,${ }^{1}$ R. Palit, ${ }^{1}$ P. Pawłowski, ${ }^{8}$ A. Pullia,${ }^{4}$ G. Raciti, ${ }^{9}$ E. Rapisarda,${ }^{9}$ H. Sann, ${ }^{1}$ C. Schwarz,${ }^{1}$ C. Sfienti, ${ }^{1}$ H. Simon,${ }^{1}$ K. Sümmerer,${ }^{1}$ M.B. Tsang, ${ }^{10}$ G. Verde, ${ }^{10}$ C. Volant,${ }^{6}$ M. Wallace,${ }^{10}$ H. Weick, ${ }^{1}$ J. Wiechula, ${ }^{1}$ A. Wieloch, ${ }^{3}$ B. Zwiegliński, ${ }^{11}$

${ }^{1}$ GSI Darmstadt, D-64291 Darmstadt, Germany

${ }^{2}$ Institut de Physique Nucléaire, $\mathrm{IN}^{2} \mathrm{P}^{3}$ et Université, F-91406 Orsay, France ${ }^{3}$ Institute of Physics, Jagiellonian University, Pl-30059 Kraków, Poland ${ }^{4}$ Istituto di Scienze Fisiche, Università and INFN, I-20133 Milano, Italy

${ }^{5}$ Institute for Nuclear Research, 117312 Moscow, Russia

${ }^{6}$ DAPNIA/SPhN, CEA/Saclay, F-91191 Gif-sur-Yvette, France

${ }^{7}$ GANIL, CEA et IN ${ }^{2} \mathrm{P}^{3}$-CNRS, F-14076 Caen, France 8 IFJ-PAN, Pl-31342 Kraków, Poland

${ }^{9}$ Dipartimento di Fisica dell'Università and INFN-LNS, I-95123 Catania, Italy ${ }^{10}$ Department of Physics and NSCL, MSU, East Lansing, MI 48824, USA 11 A. Soltan Institute for Nuclear Studies, Pl-00681 Warsaw, Poland

November 2, 2018

\begin{abstract}
Isotopic effects in projectile fragmentation at relativistic energies have been studied with the ALADIN forward spectrometer at SIS. Stable and radioactive Sn and La beams with an incident energy of $600 \mathrm{MeV}$ per nucleon have been used in order to explore a wide range of isotopic compositions. Chemical freeze-out temperatures are found to be nearly invariant with respect to the $A / Z$ ratio of the produced spectator sources, consistent with predictions for expanded systems. Consequences for the proposed interpretation of chemical breakup temperatures as representing the limiting temperatures predicted by microscopic models are discussed.
\end{abstract}




\section{Introduction}

The systematic data set on isotopic effects in spectator fragmentation collected recently at the GSI laboratory permits the investigation of the $A / Z$ dependence of the nuclear caloric curve which is of interest in several respects. It is of practical importance for isotopic reaction studies, presently conducted in many laboratories under the assumption that the basic reaction processes remain the same if only the isotopic composition of the collision partners is varied. One expects that specific effects related to the isotopic dependence of the nuclear forces can be isolated in this way[1, 2]. For example, in the statistical interpretation of isoscaling, analytic relations between the measured parameters and the symmetry term in the equation of state can be derived assuming that the freeze-out temperatures are identical for the reactions one compares[1, 3]. A significant isotopic dependence of the caloric curve would here present a complication.

The theoretical interest in the isotopic variation of breakup temperatures is motivated by the hope to establish a connection with the limiting temperatures, i.e. the maximum temperatures nuclei can sustain before they become unbound[4, 5]. Experimental information on limiting temperatures will permit tests of microscopic calculations of the nuclear equation of state at finite temperature which cannot be easily obtained by other means [6, 7]. One has to know, however, whether the observed disintegrations are primarily caused by Coulomb instabilities limiting the existence of compound nuclei or by the opening of the partition space. Since temperatures with different isotopic behaviours are predicted for these scenarios, the caloric curve can thus provide information on the reaction mechanism.

\section{$2 \quad$ Limiting temperatures}

Limiting temperatures are a manifestation of the fragility of nuclear fragments in the hot environment generated during energetic reactions. If the level of energy transfers exceeds a given limit the produced nuclei will not survive as bound objects but rather disintegrate into smaller entities, nucleons, complex particles, or very small fragments. On the other hand, for observing fragmentation, it is necessary to provide substantial amounts of energy in order to overcome the binding forces causing the resilience of nuclear systems against fragment formation [8, 9]. Multifragmentation reactions are thus characterized by a delicate energy balance.

The decay channels available for excited nuclei have been studied by Gross et al. within a phase space model[10]. Following the principles of Weisskopf evaporation theory, they calculated the asymptotic phase space available for the system. The main assumptions in this particular model were an expanded volume in order to accommodate the fragments, particle emissions faster than the reaction time so that only gamma decaying states had to be considered, and equal a priori probabilities for all accessible states. The many decay channels obtained from microcanonical sampling were sorted into the classes of evaporation, fission, cracking, and vaporization. Cracking here denotes the appearance of at least three substantial fragments while vaporization indicates the complete disintegration into nucleons and light fragments with mass numbers $A<20$. It was found that cracking is the dominant reaction channel for the studied ${ }^{238} \mathrm{U}$ nuclei at excitation energies of the order of $1 \mathrm{GeV}[10]$. Cracking diminishes quickly at smaller and higher excitation energies which reflects the above mentioned limits. The resulting rise and fall of fragment production predicted by this and other, similar, models[11] was found to be in good agreement with the experiment[12, 13].

The temperatures to be reached or exceeded for observing this new class of reaction processes have received special interest also for their connection with the nuclear equation of state. Calculations suggested a nearly linear relationship between the limiting temperatures at which compound nuclei can

still exist and the critical temperature of nuclear matter[4, 14-16]. Quantitative experimental results for the former would thus serve as a valuable source of information on nuclear matter properties. 


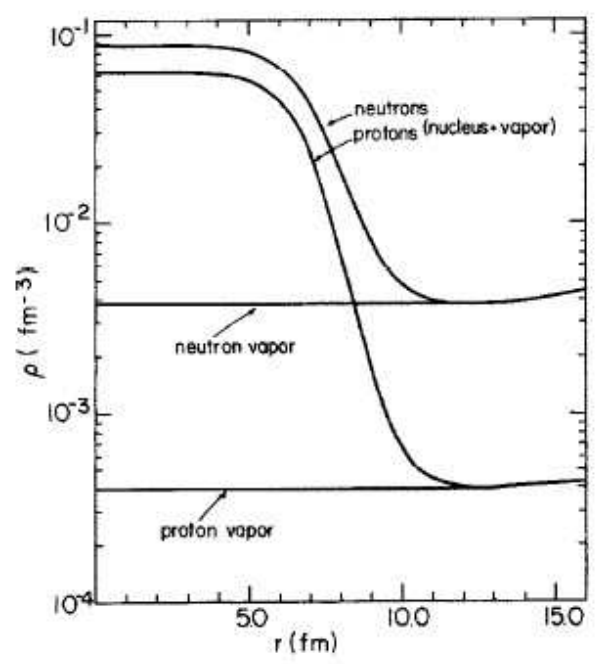

Figure 1: Radial dependence of the neutron and proton densities of nucleus+vapor and vapor solutions of the thermal Hartree-Fock equations for the uncharged ${ }^{208} \mathrm{~Pb}$ nucleus at $7 \mathrm{MeV}$ temperature and with the SKM interaction in a confining spherical box with radius $R=16 \mathrm{fm}$ (from Ref.[4]).

The stability of hot compound nuclei has been studied within the temperature-dependent HartreeFock model by several groups. The problem of accounting for the continuum components of the compound-nuclear levels has been addressed by Bonche et al. by considering the nucleus in thermal equilibrium with its surrounding vapor [4. As an example, the solution obtained for a ${ }^{208} \mathrm{~Pb}$ nucleus excited to $7 \mathrm{MeV}$ temperature is shown in Fig. 1. At temperatures exceeding this value, a self-bound solution does no longer exist, and the nuclear matter is found to be pressed against the boundaries of the confining volume of the calculation. As evident from the figure, these calculations are restricted to homogeneous matter distributions and spherical symmetry by the choice of the trial wave functions and do not explore the partition space available for fragmentation channels. The nuclear radii remain close to their ground-state values.

A particular role is played by the Coulomb force which if included drastically lowers the temperature limits in these calculations. This is best visualized in the results of Besprosvany and Levit [5] who calculated limiting temperatures for a wide variety of nuclei within a liquid-drop approximation to the Hartree-Fock theory (Fig. 2). Along the valley of stability, with decreasing mass, the effect of the decreasing $Z$ dominates over that of the increasing $Z / A$ which permits excitations to higher temperatures before the system becomes unbound. A systematic mass dependence of measured breakup temperatures in multifragmentation reactions has, therefore, led to the suggestion that they may be identified with the predicted stability limits[17, 18]. For the same reason, the limiting temperatures vary along chains of isotopes or isobars. On the proton rich side, beyond the valley of stability, they are predicted to decrease rather rapidly. These variations with mass and isospin can serve as characteristic signatures of limiting-temperature effects in experimental data.

\section{ALADIN Experiment S254}

The ALADIN experiment S254, conducted in 2003 at the SIS heavy-ion synchrotron, was designed to study isotopic effects in projectile fragmentation at relativistic energies. Besides stable ${ }^{124} \mathrm{Sn}$ and ${ }^{197} \mathrm{Au}$ beams, neutron-poor secondary Sn and La beams were used in order to extend the range of isotopic compositions beyond that available with stable beams alone (Fig. 2). The radioactive secondary beams 


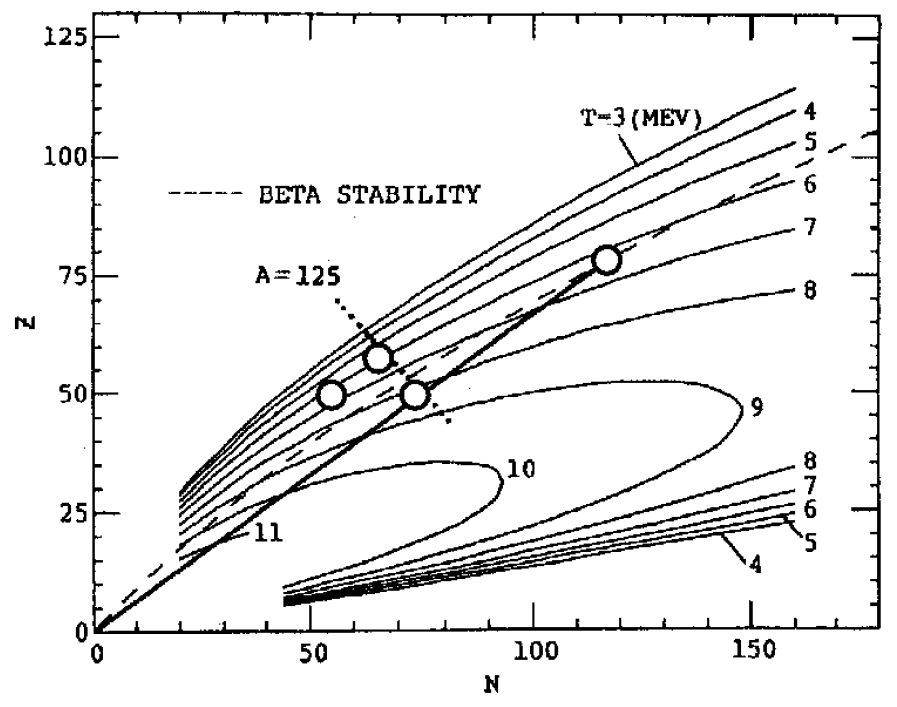

Figure 2: Location of the four projectiles used in experiment S254 in the plane of atomic number $Z$ versus neutron number $N$. The contour lines represent the limiting temperatures according to Ref. [5], the dashed line gives the valley of stability, the full line corresponds to the $N / Z=1.49$ of ${ }^{197} \mathrm{Au}$, and the dotted line marks $A=125$ (adapted from Ref.[17]).

were produced at the fragment separator FRS[19] by the fragmentation of primary ${ }^{142} \mathrm{Nd}$ projectiles of about $900 \mathrm{MeV} /$ nucleon in a thick beryllium target. The FRS was set to select ${ }^{124} \mathrm{La}$ and, in a second part of the experiment, also ${ }^{107} \mathrm{Sn}$ projectiles which were then delivered to the ALADIN setup. All beams had a laboratory energy of $600 \mathrm{MeV} /$ nucleon and were directed onto reaction targets consisting of ${ }^{\text {nat }} \mathrm{Sn}$ (or ${ }^{197} \mathrm{Au}$ for the case of ${ }^{197} \mathrm{Au}$ fragmentation).

In order to reach the necessary beam intensity of about 1000 particles/s with the smallest possible mass-to-charge ratio $A / Z$, it was found necessary to accept a distribution of neighboring nuclides together with the requested ${ }^{124} \mathrm{La}$ or ${ }^{107} \mathrm{Sn}$ isotopes. While the mass and charge of each projectile can be known from measurements with upstream detectors[20, only results obtained with the full distributions will be presented here. Their mean compositions were $\langle Z\rangle=56.8$ (49.7) and $\langle A \mid Z\rangle=$ 2.19 (2.16) for the nominal ${ }^{124} \mathrm{La}\left({ }^{107} \mathrm{Sn}\right)$ beams, respectively. Model studies consistently predict that the same $\langle A \mid Z\rangle$ values are also representative for the spectator systems emerging after the initial cascade stage of the reaction. In particular, the differences between the neutron-rich and neutron-poor cases are expected to remain the same within a few percent [3, 21].

\section{$4 \quad N / Z$ dependence of the caloric curve}

The mass resolution obtained for projectile fragments entering into the acceptance of the ALADIN spectrometer is about 3\% for fragments with $Z \leq 3$ and decreases to $1.5 \%$ for $Z \geq 6$ (standard deviations). Masses are thus individually resolved for fragments with atomic number $Z \leq 10$. The elements are resolved over the full range of atomic numbers up to the projectile $Z$ with a resolution of $\Delta Z \leq 0.2$ obtained with the TP-MUSIC IV detector[13].

Two examples of double-isotope temperatures deduced from the measured isotope yields are shown in Fig. 3 as a function of $Z_{\text {bound }}$. Besides the frequently used $T_{\mathrm{HeLi}}$ (left panel), determined from ${ }^{3,4} \mathrm{He}$ and ${ }^{6,7} \mathrm{Li}$ yields also the results for $T_{\mathrm{BeLi}}$ are displayed (right panel). For $T_{\mathrm{BeLi}}$ the isotope pairs of ${ }^{7,9} \mathrm{Be}$ and ${ }^{6,8} \mathrm{Li}$ are used which each differ by two neutrons. The double difference of their binding energies amounts to $11.3 \mathrm{MeV}$ and is nearly as large as the $13.3 \mathrm{MeV}$ in the $T_{\mathrm{HeLi}}$ case. The apparent 


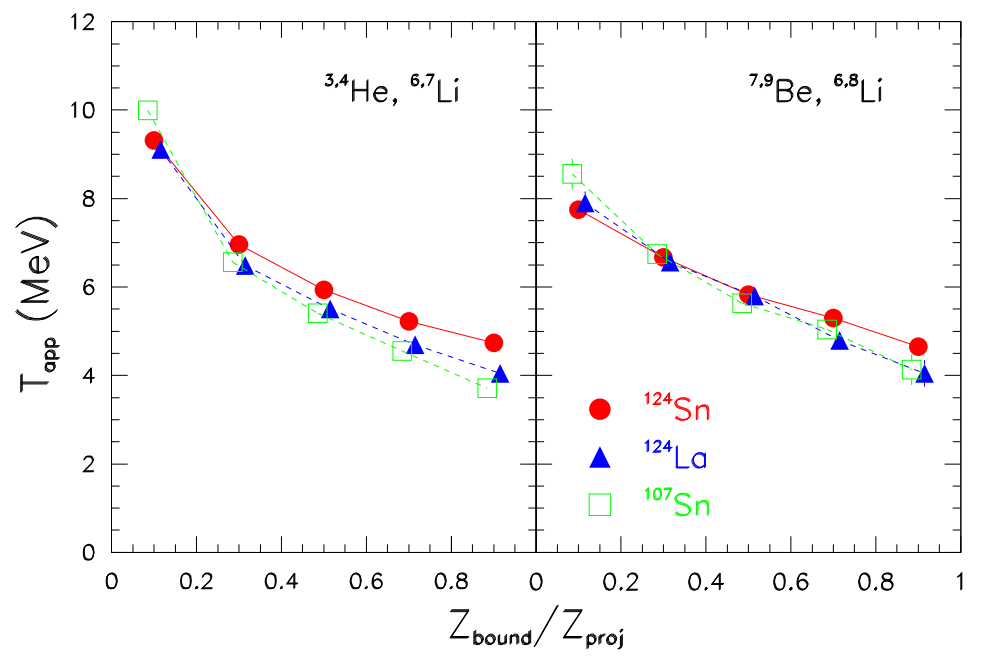

Figure 3: Apparent temperatures $T_{\mathrm{HeLi}}$ (left panel) and $T_{\mathrm{BeLi}}$ (right panel) as a function of $Z_{\text {bound }} / Z_{\text {proj }}$ for the three reaction systems produced with ${ }^{107,124} \mathrm{Sn}$ and ${ }^{124}$ La projectiles. For clarity, two of the three data sets are slightly shifted horizontally, only statistical errors are displayed.

temperatures are displayed, i.e. no corrections for secondary decays feeding the ground states of these nuclei are applied. Including such corrections will raise the temperature values by 10 to $20 \%[22,23$.

Both temperature observables show the same smooth rise with increasing centrality that was observed earlier in the study of ${ }^{197} \mathrm{Au}$ fragmentations [23, 24]. For $T_{\mathrm{BeLi}}$, the statistical uncertainties are larger in the two extreme bins at large and small $Z_{\text {bound }}$ in which intermediate-mass fragments are not abundantly produced. $T_{\mathrm{HeLi}}$, on the other hand, which mainly reflects the behavior of the ${ }^{3} \mathrm{He} /{ }^{4} \mathrm{He}$ yield ratio reaches to somewhat higher temperatures at small $Z_{\text {bound }}$. The dependence on the isotopic composition is very small and virtually non-existent in the fragmentation channels. At the larger $Z_{\text {bound }}$, for which residue production dominates, the temperatures of ${ }^{124} \mathrm{Sn}$ decays are slightly larger than those of the neutron-poor systems. This tendency is more pronounced in $T_{\mathrm{HeLi}}$ than in $T_{\mathrm{BeLi}}$.

For a quantitative comparison with the Hartree-Fock predictions, the region of transition from residue production to multifragmentation $\left(Z_{\text {bound }} / Z_{\text {proj }} \approx 0.7\right)$ seems best suited. The residue channels associated with the highest temperatures are found here, resulting from the decay of spectator systems with about $75 \%$ of the projectile mass 22]. Their limiting temperatures of $9.2 \mathrm{MeV}$ and $7.9 \mathrm{MeV}$ for the neutron-rich and neutron-poor cases, respectively, are higher than those of the nominal nuclei while their difference is somewhat smaller (cf. Fig. 2). The experimental mean values of $T_{\mathrm{HeLi}}$ and $T_{\mathrm{BeLi}}$, after applying a $20 \%$ side-feeding correction[22, 23], are $6.2 \mathrm{MeV}$ for ${ }^{124} \mathrm{Sn}$ and $5.7 \mathrm{MeV}$ for the neutron-poor systems.

The large difference in absolute magnitude between the Hartree-Fock limiting temperatures and the side-feeding corrected double-isotope temperatures are not as crucial as it may appear at first sight. As noted already by Natowitz et al.[17], the predictions depend sensitively on the type of Skyrme force used in the calculations [15, 16]. A linear rescaling with a factor 0.71, approximately corresponding to the results obtained by Song and $\mathrm{Su}$ with the SkM* force 15 leads to the overall best agreement in absolute magnitude. The corresponding critical temperature for infinite nuclear matter is $T_{c}=15 \mathrm{MeV}$. However, even on the reduced scale, the predicted $N / Z$ dependence, $\Delta T \approx 0.9 \mathrm{MeV}$ for the studied systems, is considerably larger than the experimental value. Furthermore, the interpretation of the $Z_{\text {bound }}$ dependence as being caused by the mass dependence[17] would require that the temperature difference remains on a similar level for the full range of $Z_{\text {bound }} / Z_{\text {proj }}<0.7$ which is not observed. 


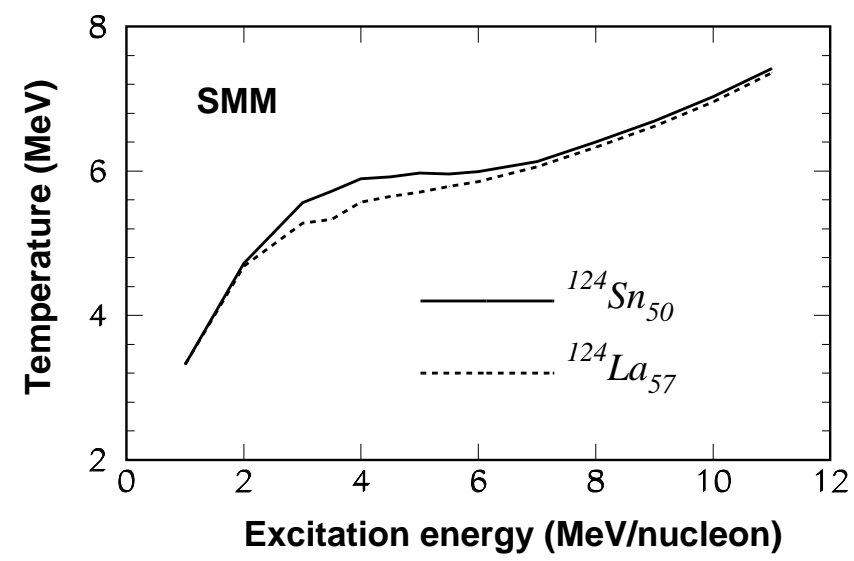

Figure 4: Caloric curves for ${ }^{124} \mathrm{Sn}(Z=50)$ and ${ }^{124} \mathrm{La}(Z=57)$ as calculated with the Statistical Multifragmentation Model (from Ref. [28]).

A much reduced role for Coulomb effects is predicted by models which include expansion[25, 26] or which allow the partitioning of the system. As noted long ago by Barz et al.[27], the limiting temperatures emerging from the Hartree-Fock approach are systematically too high because they are not going beyond the mean-field approximation and the formation of clusters in the excited medium is not considered. The Coulomb forces act with reduced strength in the expanded volume and are partly compensated by the nuclear forces within the formed fragments. Calculations performed more recently[28] with the Statistical Multifragmentation Model[11] predict much smaller differences for the breakup temperatures of neutron-rich and neutron-poor systems with a dependence on the excitation energy that is in very good agreement with the experimental data (Fig. 44).

The open question that remains to be answered concerns the nature of the instability driving the system into the expanded configurations sampled in the statistical approaches. Following the statistical principles cited by Gross et al.[10], the mere opening of the asymptotic phase space will suffice, leading to a phase-space driven instability rather than the Coulomb instability appearing from the HartreeFock simulations. Furthermore, taking recourse to recent dynamical studies, one observes that the partitioning of the system with a loss of binding between the emerging parts may occur rather early in the collision[29].

\section{Summary}

The temperatures limiting the existence of self-bound nuclei or nuclear fragments appear in different roles in fragmentation reactions. The limits have to be overcome to initiate the disintegration of the produced excited systems while they should not be exceeded by the fragments to be observed. A quantitative knowledge of these limits is not only of interest for the understanding of the reaction mechanisms involved but also for the more fundamental reason that they can provide information on the nuclear-matter equation-of-state. Hartree-Fock calculations predict a linear relation between the limiting temperatures of excited compound nuclei and the critical temperature limiting the liquid-gas phase transition of nuclear matter.

The proposed interpretation of breakup temperatures measured in spectator fragmentation as representing the limiting temperatures was derived from a common mass dependence. This is only partially confirmed by the new experimental data obtained from the study of isotopic effects in spectator fragmentation. The temperatures are smaller than the general level of the predictions and, in particular, 
their variation with $N / Z$ is smaller than expected. The latter result, on the other hand, is compatible with the assumption of identical reaction trajectories usually made in isotopic reaction studies. From the highest temperatures observed for compound channels, a critical temperature of about $15 \mathrm{MeV}$ is deduced. It should be considered as a lower limit because phase-space driven instabilities may initiate the disintegration before the static compound limit is reached. The good agreement with predictions of the Statistical Fragmentation Model supports this assumption.

This work has been supported by the European Community under contract No. HPRI-CT-199900001 and by the Polish Ministry of Science and Higher Education under Contracts No. 1 P03B 105 28 (2005 - 2006) and N202 $16032 / 4308$ (2007-2009).

\section{References}

[1] M. Colonna and M.B. Tsang, Eur. Phys. J. A 30 (2006) 165, and references therein

[2] Bao-An Li, Lie-Wen Chen, and Che Ming Ko, Phys. Rep. 464 (2008) 113

[3] A.S. Botvina, O.V. Lozhkin, and W. Trautmann, Phys. Rev. C 65 (2002) 044610

[4] P. Bonche, S. Levit, and D. Vautherin, Nucl. Phys. A 436 (1985) 265

[5] J. Besprosvany and S. Levit, Phys. Lett. B 217 (1989) 1

[6] M. Baldo, L.S. Ferreira, and O.E. Nicotra, Phys. Rev. C 69 (2004) 034321

[7] P. Wang et al., Nucl. Phys. A 748 (2005) 226

[8] B. Tamain, Eur. Phys. J. A 30 (2006) 71

[9] M. Colonna, J. Cugnon, and E.C. Pollacco, Phys. Rev. C 55 (1997) 1404

[10] D.H.E. Gross, Zhang Xiao-ze, and Xu Shu-yan, Phys. Rev. Lett. 56 (1986) 1544

[11] J.P. Bondorf et al., Phys. Rep. 257 (1995) 133

[12] C.A. Ogilvie et al., Phys. Rev. Lett. 67 (1991) 1214

[13] A. Schüttauf et al., Nucl. Phys. A 607 (1996) 457

[14] G. Sauer, H. Chandra, and U. Mosel, Nucl. Phys. A 264 (1976) 221

[15] H.Q. Song and R.K. Su, Phys. Rev. C 44 (1991) 2505

[16] A. Kelić, J.B. Natowitz, and K.-H. Schmidt, Eur. Phys. J. A 30 (2006) 203

[17] J.B. Natowitz et al., Phys. Rev. C 52 (1995) R2322

[18] J.B. Natowitz et al., Phys. Rev. C 65 (2002) 034618

[19] H. Geissel et al., Nucl. Instr. and Meth. B 70 (1992) 286

[20] J. Łukasik et al., Nucl. Instr. and Meth. A 587 (2008) 413

[21] A. Le Fèvre et al., Phys. Rev. Lett. 94 (2005) 162701

[22] J. Pochodzalla et al., Phys. Rev. Lett. 75 (1995) 1040

[23] W. Trautmann et al., Phys. Rev. C 76 (2007) 064606

[24] Hongfei Xi et al., Z. Phys. A 359 (1997) 397 ; erratum in Eur. Phys. J. A 1 (1998) 235

[25] C. Hoel, L.G. Sobotka, and R.J. Charity, Phys. Rev. C 75 (2007) 017601

[26] S.K. Samaddar et al., Phys. Rev. C 76 (2007) 041602(R)

[27] H.W. Barz, J.P. Bondorf, and H. Schulz, Phys. Lett. B 184 (1987) 125

[28] R. Ogul and A.S. Botvina, Phys. Rev. C 66 (2002) 051601(R)

[29] A. Le Fèvre and J. Aichelin, Phys. Rev. Lett. 100 (2008) 042701 